\title{
Non-Volatile Magnetic Memory Combined with AND/NAND Boolean Logic Gates Based on Geometry-Controlled Magnetization Switching
}

\author{
Ziyao Lu ${ }^{1}$, Chengyue Xiong ${ }^{1}$, Hongming $\mathrm{Mou}^{1}$, Zhaochu Luo ${ }^{2}$, Wanjun Jiang ${ }^{3}$, Xixiang Zhang ${ }^{4}$, \\ and Xiaozhong Zhang ${ }^{1 *}$ \\ 1. School of Materials Science and Engieering and Key Laboratory of Advanced Materials (MOE), Tsinghua University, Beijing \\ 100084, China. \\ 2. Department of Materials, ETH Zurich, 8093 Zurich, Switzerland \\ 3. Department of Physics, Tsinghua University, Beijing 100084, China \\ 4. Division of Physical Science and Engineering, King Abdullah University of Science and Technology (KAUST), Thuwal 239955, \\ Kingdom of Saudi Arabia
}

Received 1 Apr 2020, revised 15 Apr 2020, accepted 20 Apr 2020, published 1 Jun 2020, current version 15 Jun 2020 . (Dates will be inserted by IEEE; "published" is the date the accepted preprint is posted on IEEE Xplore ${ }^{\circledR}$; "current version" is the date the typeset version is posted on Xplore ${ }^{\circledR}$.

\begin{abstract}
Spin-orbit torque(SOT) has been widely used in data writing of spintronic memory devices by currentinduced magnetization switching. The typical structure of SOT-induced magnetization switching of ferromagnetic multilayers with perpendicular magnetic anisotropy(PMA) such as $\mathrm{Ta} / \mathrm{CoFeB} / \mathrm{MgO}$ allowed the ferromagnetic and adjacent nonmagnetic layer to be patterned independently. Here, we studied the role of device geometry in the manipulation of magnetization switching by placing two separated CoFeB magnetic bits at different locations on Ta layer with trapezoid shape. Manipulation of the magnetization states of the magnetic bits was achieved simply by applying different write current. Both memory writing and Boolean logic functions AND/NAND with large logic output ratio have been demonstrated experimentally.
\end{abstract}

\section{INTRODUCTION}

In conventional computers, memory based on non-volatile magnetic bits and processor based on Complementary Metal-OxideSemiconductor (CMOS) logic gates are separate. Such separation causes computers to suffer from von Neumann performance bottleneck. Because frequent data transfer is required between memory and processor, a huge amount of time and energy is consumed [Backus 1983, Liu 2005], which is disadvantageous for pursuing faster speed and lower power consumption. To break through the von Neumann bottleneck, research on logic-in-memory architecture has been intensively conducted. Benefiting from the non-volatility of ferromagnetic magnetization and the rich functionality provided by emerging magnetoelectric transport phenomenon, spintronic device has become one of the most competitive candidate of future non-von Neumann computer architecture [Makarov 2016]. For spintronic devices combining logic with memory, data is stored and processed in the form of electron spins. Hence, electric manipulation of spins becomes one of the most important issues for spintronic data writing. Among all the widely-studied methods of magnetization switching, manipulation of spins driven by spin-orbit torque (SOT) has drawn much attention due to the potential low power consumption and fast device operation speed. In the prototype of SOT-based spintronic devices, spin current generated from the spin-orbit interaction in an nonmagnetic (NM) layer is utilized to switch the magnetization direction in an adjacent ferromagnetic (FM) layer when an in-plane electric current is applied in the NM layer [Manchon 2019, Kato 2018]. Compared with other commonly used methods of

Corresponding author: Xiaozhong Zhang (xzzhang@tsinghua.edu.cn). Digital Object Identifier: 10.1109/LMAG.XXXX.XXXXXXX (inserted by IEEE). magnetization manipulation such as spin transfer torque (STT), there could be more freedom in the design of SOT-based devices, which makes it possible to manipulate the performance of the device by geometrical design [Safeer 2016]. In SOT-based devices, the NM layer and FM layer could be patterned independently with different size and shape. The change of shape of NM layer could lead to different distribution of current density. Because the spin orbit torque exerted on the electron spin in the FM layer was related to the current density of the NM layer, manipulation of the magnetization direction of the FM layer would be dependent on its position on the NM layer. It has been reported that specific patterning of both FM layer and NM layer could be utilized to control the magnetization state of the FM layer [Safeer 2016, Siddiqui 2020, Martinez 2016, Wang 2019]. Hence, the feasibility of applying such method in spinbased data storage has been confirmed. However, in the field of data processing, especially in spin-based Boolean logic, the potential of geometry-assisted manipulation of spins still remains to be exploited. Here, we propose a new magnetic logic-in-memory device combining manipulation of magnetic spins with magnetic Boolean logic gate of AND and NAND. The logic inputs were stored in the form of magnetization of two separate FM bits located on one NM layer. By designing the shape of the NM layer, the data stored in the two FM bits was written independently by applying current in the NM layer with the assistance of a constant external magnetic field and logic operation could be performed based on the accumulative effect of the anomalous Hall effect (AHE) of the two magnetic bits. Since the magnetic response caused by AHE was not large enough to ensure the reliability of logic operations, the AHE-induced magnetic response was coupled with the nonlinear transport characteristics of semiconductor elements with negative differential resistance (NDR). It has been reported that introducing NDR elements to logic devices based on AHE could magnify the logic 
output of the device [Luo 2017, Singh 2019, Pu 2020]. In our device, the logic output ratio of the device reached $5 \times 10^{3} \%$ benefitting from the NDR-induced enhancement of AHE.

\section{EXPERIMENT}

Magnetic multilayer Ta $(3 \mathrm{~nm}) / \mathrm{Co}_{40} \mathrm{Fe}_{40} \mathrm{~B}_{20}(0.7 \mathrm{~nm}) / \mathrm{MgO}(1 \mathrm{~nm})$ with $5 \mathrm{~nm}$ thick $\mathrm{SiO}_{2}$ capping layer was used to fabricate our spintronic device. The layer thickness in the parentheses were nominal thickness deduced from the deposition rate. The multilayer was deposited on thermally oxidized $\mathrm{Si}$ substrate with $\mathrm{SiO}_{2}$ thickness of $300 \mathrm{~nm}$ by sputtering (Rotaris, Singulus) with base pressure $<8.0 \times 10^{-9}$ mbar. Perpendicular magnetic anisotropy was achieved after annealing at $220^{\circ} \mathrm{C}$ in vacuum $\left(<2 \times 10^{-4} \mathrm{~Pa}\right)$ for 1 hour. The device was patterned by UV lithography and Ar-ion milling, and $\mathrm{Ti}(10 \mathrm{~nm}) / \mathrm{Au}(50 \mathrm{~nm})$ was used as electrodes. The magnetic transport measurements, including manipulation of magnetization direction of the $\mathrm{CoFeB}$ layer and experimental demonstration of magnetic logic functions, were conducted using Keithley 2400 and Keithley 2182A at room temperature.

\section{RESULTS AND DISCUSSION}

The structure of the device was shown in Fig.1. The $\mathrm{Ta} / \mathrm{CoFeB} / \mathrm{MgO}$ multilayer with $\mathrm{PMA}$ was firstly patterned in trapezoidal shape. Then in the region marked with blue color in Fig. 1, Ar-ion milling was used to etch the film to the bottom Ta layer. As a result, current could still be applied in the bottom Ta layer, whereas the top magnetic layer was separated into two bits. The separated magnetic bits could not only store binary data in the form of ferromagnetic spins, but also serve as logic inputs for Boolean logic gates. Because the two bits were separated by Ar-ion milling in the blue region in Fig. 1(a), their magnetization states could be manipulated individually by spin-orbit torque generated from inplane current in the Ta layer. Due to the trapezoidal shape, the width of the Ta layer increased gradually from the left side to the right side. Hence, when current was applied between left and right electrodes, the current density in the Ta layer beneath the left magnetic bit $\mathrm{P}$ was greater than that of magnetic bit $\mathrm{Q}$ and larger torque was exerted to the magnetic spins in bit $\mathrm{P}$ because of higher current density. Hence, with the assistance of an external in-plane magnetic field $B_{x}$ of $-3 \mathrm{mT}$ colinear with the applied current, the critical current value to switch the magnetization direction of magnetic bit $P$ would be smaller than bit $\mathrm{Q}$. Such difference in the critical switching current of the two bits resulted from the trapezoidal shape of the Ta layer, and could be utilized to manipulate the magnetization states of the magnetic bits. As shown in Fig.1(b), by applying write current pulse between left and right electrodes with magnitude ranging from $-5 \mathrm{~mA}$ to $5 \mathrm{~mA}$, four different levels of anomalous Hall resistance measured by read current pulses $I_{\text {Read }}$ of $0.05 \mathrm{~mA}$ applied after each write pulse was detected. The four different levels of $R_{x y}$ were in accordance with the four possible combinations of the magnetization states of the two bits $(\uparrow \uparrow)(\uparrow \downarrow)(\downarrow \uparrow)$ and $(\downarrow \downarrow)$. If we define “ $\uparrow$ " (“ $\downarrow$ ”) magnetization direction as binary number " 0 "("1"), the device could store two bits of binary data, indicating that our device with trapezoidal geometrical design was capable of writing and storing two digits of data in the two magnetic bits. It should be noted that although the width of Ta layer under each magnetic bit varied continuously because of the trapezoidal shape, no other intermediate resistance states were found in our measurements of $R_{x y}$. Such result could be explained by different critical current for domain wall nucleation and propagation. If the former was larger than the latter, domain wall would propagate immediately after the nucleation took place and no intermediate states could be detected, as reported by Zhang et al in $\mathrm{Ta} / \mathrm{CoFeB} / \mathrm{MgO}$ system [Zhang 2015].

The anomalous Hall measurement had proved that all four combinations of the magnetization states of the two magnetic bits in our device could be achieved. However, to prove that the difference in critical switching current of the two bits was resulted from the difference of current density caused by the shape of the Ta layer, direct evidence was still needed to prove that the magnetic bit with narrower Ta layer underneath was always switched first. Magnetooptical Kerr effect (MOKE) was therefore used for in-situ observation of the change of magnetization direction of the magnetic bits when current and external magnetic field was applied.

As shown in MOKE images (Fig. 2), when constant external inplane magnetic field was applied, the magnetization direction of both magnetic bits could be switched from one direction to the other, depending on the direction of applied write current. No matter whether the magnetization was switched from upward to downward or vice versa, the current needed to switch the magnetic bit on the left side was always smaller than the one on the right. Because the bottom Ta layer was narrower on the left and wider on the right. So the current density in the Ta layer would be larger on the left side than the right side. This made the current required to reverse the magnetization direction of the left bit lower than the right bit. These results from MOKE confirmed that the sequence of magnetization switching of the two magnetic bits depended on the width of Ta layer underneath., which supported our presumption that the different critical switching current of the two magnetic bits was caused by the shape of the device. Furthermore, since all four possible magnetization states $(\uparrow \uparrow)(\uparrow \downarrow)(\downarrow \uparrow)$ and $(\downarrow \downarrow)$ were presented in the MOKE images, the capability of our device to store two digits of binary data in separated memory bits by stepwise switching has been proved. The different value of critical switching current between MOKE experiment and anomalous Hall measurement could be explained by different modes of writing pulse. In AHE measurement, the write and read pulses were applied to the device continually, whereas in MOKE measurement, discrete write pulses with different magnitude were used to drive the magnetization reversal. As a result, heating effect in the AHE measurement would be more obvious than MOKE measurement, which could account for the smaller switching current in the AHE experiment than MOKE.

Apart from memory, the magnetization states of the two bits could also serve as logic inputs of Boolean logic gates. Due to the anomalous Hall effect, conducting electrons with opposite spins directions tended to be deflected into different directions. Thus, when electrical current flew into the device from top electrode, and out of the device from left and right side (denoted as left and right channel), as shown in Fig. 3(a), the change of magnetization direction of the magnetic bits would lead to change in the magnitude of the channel current. When the magnetization direction of the FM layer was reversed from downward to upward, the current would tend to decrease in the left channel and increase in the right channel and vice versa. Such magnetic response of electric current could lead to imbalance of left and right current channels, and could be utilized 
for conducting Boolean logic operations. However, for the $\mathrm{Ta} / \mathrm{CoFeB} / \mathrm{MgO}$ multilayer used in the device, the change of channel current in response to magnetization switching was not large enough to achieve large enough ON/OFF logic output ratio for reliable logic operations. In order to get large logic ON/OFF ratio, the magnetic response of channel current needed to be enlarged. To solve this problem, nonlinear enhancement method induced by nonlinear elements with negative differential resistance (NDR) was applied. Introduction of NDR components could lead to bifurcation of output current and increase the ON/OFF ratio of logic output [Luo 2017]. The logic circuit with NDR elements connected to the trapezoidshaped device was shown in Fig. 3(a). Measurement result of output current in both channels with different logic inputs showed that bifurcation of current magnitude in left and right channels appeared and the ratio of high and low output current was enlarged (Fig. 3(b)). If large output current was defined as logic output "1" and small output current as "0", our device could simultaneously perform AND Boolean logic operation in the left channel and NAND

Boolean logic operation in the right channel with logic output of $5 \times$

$10^{3} \%$. Such high output ratio was important to meet the requirement of stability for Boolean logic gates (Table 1). For proof-of-concept, the NDR elements used in the following experiment were composed of commercial semiconductor elements considering simplicity and stability. For better performance, other materials could also be used to fabricate NDR elements with faster speed and lower energy consumption such as phase change materials with insulator-to-metal transition [Pu 2020].

The mechanism of the enhancement of ON/OFF ratio due to the nonlinear $I-V$ characteristics of NDR elements could be explained via load-line analysis of the circuit. When the device with magnetic bits was used as logic inputs (Fig. 4(a)), due to the linear $I-V$ characteristics, it could be modeled as a Y-type resistor network consisted of three resistors $R_{T o p}, R_{\text {Left }}$, and $R_{\text {Right }}$, and the anomalous Hall effect was described as the dependence of the resistance value of $R_{\text {Left }}$ and $R_{\text {Right }}$ on the magnetization status of the magnetic bits. Change of resistance of $R_{\text {Left }}$ and $R_{\text {Right }}$ due to magnetization switching would lead to change of output current in left and right

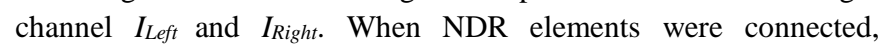
according to Kirchhoff's Law, the following relations should be followed for the circuit: $V_{0}=I_{\text {Left }} R_{\text {Left }}+V_{\text {Left }}^{\text {NDR }}=I_{\text {Right }} R_{\text {Right }}+V_{\text {Right }}^{\text {NDR }}$. In addition, the relation of $V_{\text {Left }}^{N D R}\left(V_{R i g h t}^{N D R}\right)$ with $I_{\text {Left }}\left(I_{\text {Right }}\right)$ was determined by the $I-V$ characteristics of the NDR element. Hence, for each output channel, if we superimpose the S-shape $I$ - $V$ curve of the NDR element with the straight line (referred as load line) $V^{N D R}=V_{0}-I R$, the current $I$ and voltage $V_{0}$ could be calculated by the coordinates of two critical points: the intersection point of the two curves $\left(V^{N D R}, I\right)$ (denoted as working point in the following texts), and the intersection point of the load line with $\mathrm{X}$ axis $\left(V_{0}, 0\right)$. Because the $I-V$ characteristics of NDR and the value of $V_{0}$ were the same for both output channels, as source current $I_{0}=I_{\text {Left }}+I_{\text {Right }}$ increased, the trend of channel current $I_{\text {Left }}$ and $I_{\text {Right }}$ could be directly read out from the motion of the working points of left and right channels (Figure 3(b)). Considering the case when the magnetization direction of both magnetic bits pointed upward, because of the AHE of the magnetic bits, the effective resistance of left channel $R_{\text {Left }}$ was larger than that of right channel $R_{\text {Right }}$, resulting in smaller slope of load line representing the left channel. When the source current was small, the two load lines both intersected with the S-shape curve at the first region of the S-shape $I-V$ curve of the NDR element, where the differential resistance was positive. As $I 0$ increased, the working points would gradually shift from left to right until the load line of the right channel was tangent to the $I-V$ curve of NDR element. Because the load line of right channel had larger slope, the working point of right channel would firstly move into the negative differential resistance region of the S-shape $I$ - $V$ curve. As the current in the right channel $I_{\text {Right }}$ increased as the working point of right channel moved upward in the negative differential resistance region, both load lines would shift leftward and $V_{0}$ would decrease. Since the working point of left channel still located in the positive differential resistance region, the left shift of load line would result in decrease of output current $I_{\text {Left. }}$. As a result, the output current of right channel would rise rapidly with source current while the current in left channel would decrease, which accounted for the large bifurcation of current between left and right channel in our experiment. Such load line analysis method could be adapted for the other magnetization states $(\downarrow, \downarrow)(\uparrow, \downarrow)$ and ( $\downarrow$, $\uparrow)$ (Fig. 4(c)). Thus, the magnitude of channel current was determined by the magnetization states of the magnetic bits, and the difference between high and low output current could be enhanced by the bifurcation of channel current caused by NDR elements. Hence, our device was capable of performing Boolean logic operations AND/NAND simultaneously with large ON/OFF ratio.

It has been experimentally demonstrated that our device was capable of nonvolatile data storage together with performing Boolean logic operations. For industrial application aspects, the scalability, energy efficiency and reliability need to be considered. For scalability, the geometry-assisted magnetization manipulation of magnetic bits allows multiple bits to share one write path. By controlling the distribution of current density in the NM layer by designing the shape of the NM layer, the magnetization direction of the magnetic bits integrated on the NM layer coupling could be manipulated. Thus, compared with the case where each magnetic memory bit occupied a whole stack, the geometry-assisted manipulation of multiple magnetic bits sharing the same write line allows multiple bits of data to be stored in one stack. Because no complex external electric connections is introduced, the integration of magnetic bits consumes less space. Because any two adjacent bits could be used as the logic inputs for Boolean logic operations, the reduction of space occupation beneficial for achieving the combination of logic and memory with higher storage density. It should be noted that the scaling down of the device raises high requirements for the accuracy of lithography in the aspect of logic functions. Because misalignment of the magnetic bits and the NM layer would introduce errors to the AHE-induced current imbalance between left and right channels and cause wrong logic outputs. Such problem could be alleviated by using magnetic materials with larger anomalous Hall resistance such as ferrimagnetic Heusler alloy [Zhao 2019, Finley 2019]. The energy consumption of the device for performing one logic operation is related to the speed of both magnetic element and NDR. In the above experimental demonstration of logic functions, the S-type NDR elements were consisted of bipolar junction transistor pairs with operation speed of $\sim 300$ ns, which was much slower than the speed of magnetic 
element. Thus, the speed of the device was limited by the NDR and the energy cost for one logic operation was $\sim 30 \mathrm{pJ}$. However, it has been proved that the S-type NDR could be replaced with insulatorto-metal transition (IMT) materials such as $\mathrm{VO}_{2}$ to decrease the time delay on the NDR [Pu 2020]. If $\mathrm{VO}_{2}$ is used for the NDR elements, the speed of NDR could be reduced to $\sim 7 \mathrm{~ns}$ and the energy consumption could be reduced to $\sim 0.7 \mathrm{pJ}$. Moreover, compared with CMOS-based logic devices, the nonvolatile logic-in-memory property of our device could save more time and energy. On one hand, instant-on functionality and zero quiescent power consumption is achieved owing to the nonvolatility. On the other hand, because the stored data could be directly used for Boolean logic operations, the time and energy consumed in the data transmission process between memory and logic units in conventional von Neumann architecture could be saved. For reliability, in the aspect of a single device, the large logic ON/OFF ratio resulting from the coupling of anomalous Hall effect and NDR effect indicates potential high reliability and low error rate. Moreover, in our device, the $\mathrm{MgO}$ layer takes less damage compared to MTJ-based devices because electric current does not need to flow through the $\mathrm{MgO}$ layer in logic or memory process. In the aspect of device-device variations, compared with MTJ-based devices, our device has advantage in two aspects. Firstly, the logic function of our device is based on the AHE of the magnetic film and the I-V characteristics of NDR element instead of the TMR effect for MTJbased devices. Secondly, the logic output ratio of our device is much larger than the TMR value of MTJs. These two factors make our device free from the problem of device-device variation of TMR values for MTJ-based devices. It should be noted that there are still other threats to the uniformity of devices caused by the limitation of processing accuracy. As mentioned above, such limitation is also disadvantageous for the scaling of the device and it is thus expected that usage of materials with larger anomalous Hall resistance could alleviate both problems.

In conclusion, by combining geometrical design with magnetization reversal driven by spin-orbit torque, manipulation of magnetization states of storage bits integrated on the same write line was realized together with Boolean logic operation, indicating the potential of our device in non-von Neumann architecture. The important role of geometrical design in our device proved that geometry tuning could be a powerful tool in device design to improve the performance and develop new functions of spintronic devices .

\section{ACKNOWLEDGMENT}

This work was sponsored by National Key R\&D Program of China (Grant No.: 2017YFA0206202) and National Science Foundation of China (Grant No.: 11674190).

\section{REFERENCES}

Backus J (1983), "Can programming be liberated from the von Neumann style? A functional style and its algebra of programs," in Programming languages: A grand tour, Rockville, MD, USA: Computer Science Press, pp. 146-174.

Liu C C, Ganusov I, Burtscher M, Tiwari S (2005), "Bridging the processor-memory performance gap with 3D IC technology," IEEE Des. Test Comput., vol. 22, pp. 556-564, doi: $10.1109 / \mathrm{mdt} .2005 .134$.

Makarov A, Windbacher T, Sverdlov V, Selberherr S (2016), "CMOS-compatible spintronic devices: a review," Semicond. Sci. and Technol, vol. 31, pp. 113006, doi: 10.1088/0268-1242/31/11/113006.

Manchon A, Železný J, Miron I M, Jungwirth T, Sinova J, Thiaville A, Garello K,
Gambardella P (2019), "Current-induced spin-orbit torques in ferromagnetic and antiferromagnetic systems," Rev. Mod. Phys., vol. 91, pp. 035004, doi: 10.1103/RevModPhys.91.035004.

Kato Y, Saito Y, Yoda H, Inokuchi T, Shirotori S, Shimomura N, Oikawa S, Tiwari A, Ishikawa M, Shimizu M, Altansargai B, Sugiyama H, Koi K, Ohsawa Y, Kurobe A (2018), "Improvement of Write Efficiency in Voltage-Controlled Spintronic Memory by development of a Ta-B Spin Hall Electrode," Phys. Rev. Appl., vol. 10, pp. 044011, doi: 10.1103/PhysRevApplied.10.044011.

Safeer C K, Jué E, Lopez A, Buda-Prejbeanu L, Auffret S, Pizzini S, Boulle O, Miron I M, Gaudin G (2016), "Spin-orbit torque magnetization switching controlled by geometry," Nat. Nanotechnol., vol. 11, pp. 143-146, doi: 10.1038/nnano.2015.252.

Siddiqui S A, Dutta S, Tang A, Liu L, Ross C A, Baldo M A (2020), "Magnetic Domain Wall Based Synaptic and Activation Function Generator for Neuromorphic Accelerators," Nano Lett., vol. 20, pp. 1033-1040, doi: 10.1021/acs.nanolett.9b04200.

Martinez E, Alejos O, Hernandez M A, Raposo V, Sanchez-Tejerina L, Moretti S (2016), "Angular dependence of current-driven chiral walls," Appl. Phys. Express, vol. 9, pp. 4, doi: 10.7567/apex.9.063008.

Wang Z, Li Z, Wang M, Wu B, Zhu D, Zhao W (2019), "Field-free spin-orbit-torque switching of perpendicular magnetization aided by uniaxial shape anisotropy," Nanotechnol., vol. 30, pp. 375202, doi: 10.1088/1361-6528/ab2831.

Luo Z C, Lu Z Y, Xiong C Y, Zhu T, Wu W, Zhang Q, Wu H Q, Zhang X X, Zhang X $Z$ (2017), "Reconfigurable Magnetic Logic Combined with Nonvolatile Memory Writing," Adv. Mat., vol. 29, pp. 6, doi: 10.1002/adma.201605027.

Pu Y, Mou H, Lu Z, Nawaz S, Wang G, Zhang Z, Yang Y, Zhang X, Zhang X (2020), "Speed enhancement of magnetic logic-memory device by insulator-to-metal transition," Appl. Phys. Lett., vol. 117, pp. 022407, doi: 10.1063/5.0013301.

Zhang C, Fukami S, Sato H, Matsukura F, Ohno H (2015), "Spin-orbit torque induced magnetization switching in nano-scale $\mathrm{Ta} / \mathrm{CoFeB} / \mathrm{MgO}$," Appl. Phys. Lett., vol. 107, pp. 012401, doi: 10.1063/1.4926371.

Singh R, Luo Z, Lu Z, Saleemi A S, Xiong C, Zhang X (2019), "Thermal stability of NDR-assisted anomalous Hall effect based magnetic device," J. Appl. Phys., vol. 125, pp. 203901, doi: 10.1063/1.5088916

Zhao X P, Lu J, Mao S W, Yu Z F, Wei D H, Zhao J H (2019), "Spin-orbit torque induced magnetization switching in ferrimagnetic Heusler alloy D0(22)-Mn3Ga with large perpendicular magnetic anisotropy," Appl. Phys. Lett., vol. 115, pp. 5, doi: $10.1063 / 1.5125675$.

Finley J, Lee C H, Huang P Y, Liu L Q (2019), "Spin-Orbit Torque Switching in a Nearly Compensated Heusler Ferrimagnet," Adv. Mat., vol. 31, pp. 6, doi: 10.1002/adma.201805361. 

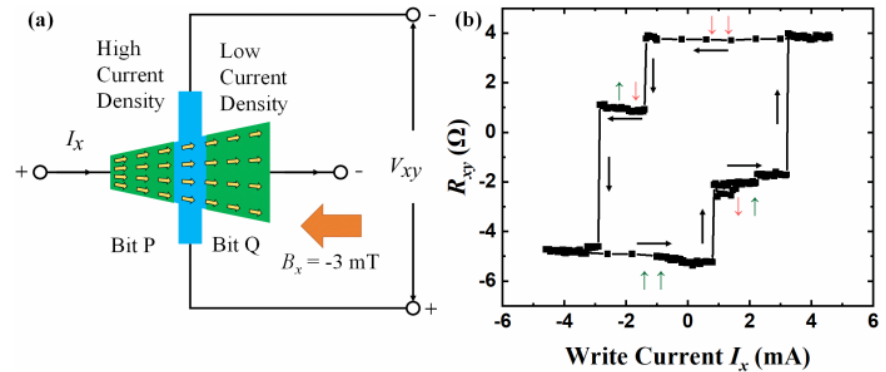

Fig. 1 Schematics of device structure and current-driven manipulation of magnetization states. a. When write current $I_{x}$ was applied, the trapezoid-shaped Ta layer would cause the current density to decrease from left to right side. With external magnetic field $B_{x}=-3 \mathrm{mT}$, such change in current density would lead to different switching current of magnetic bit $\mathrm{P}$ and $\mathrm{Q}$. b. Four different stages of $R_{x y}=V_{x y} / I_{\text {Read }}$ was detected when write current was swept between $-5 \mathrm{~mA}$ to $+5 \mathrm{~mA}$, indicating four different combinations of magnetization directions of bit $\mathrm{P}$ and $\mathrm{Q}$, which was represented by the four groups of arrows next to each stage of $R_{x y}$ in the figure. In each group with two arrows, the left arrows represented the magnetization direction of bit $\mathrm{P}$ and the right arrows represented the magnetization direction of bit $\mathrm{Q}$. The green and red color of the arrows represented magnetization up and down respectively.

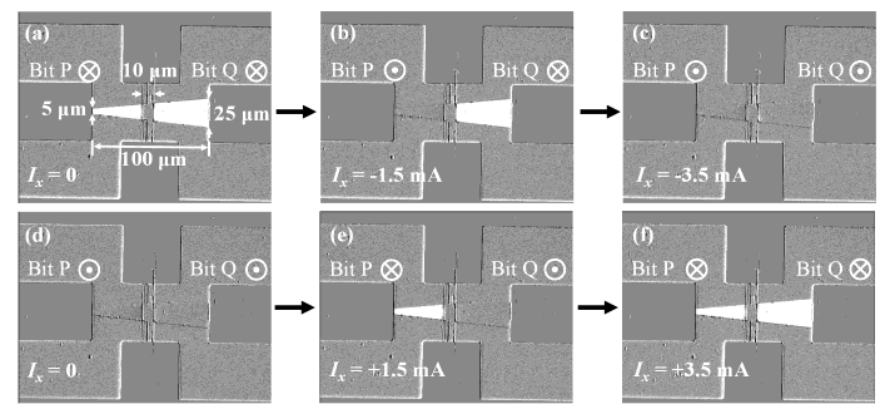

Fig. 2 MOKE images of the device. The magnetization direction of the two separated trapezoid-shape magnetic bits could be observed directly by the color, where grey and white represented magnetization upward and downward, respectively. The device was placed under a constant external in-plane magnetic field $B_{x}=-3 \mathrm{mT}$. The initial magnetization state of the device when $I_{x}=0$ was saturated by an out-of-plane magnetic field, which was removed before $I_{x}$ was applied to the device. a-c. Both magnetic bits were initially magnetized downward and switched to upward by negative $I_{x}$. d-f. Both magnetic bits were initially magnetized upward and switched to downward by positive $I_{x}$. In both cases, the current needed to switch the smaller bit $\mathrm{P}$ was smaller than that of the larger bit Q.

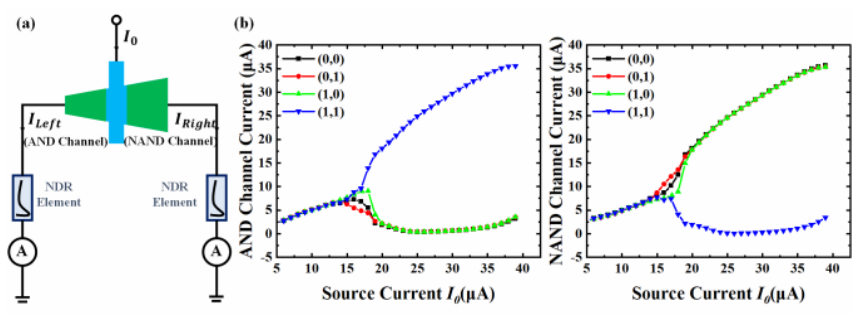

Fig. 3 Device setup and experiment result of Boolean logic operation AND and NAND. a. Schematics of logic circuit. The two magnetic bits in the trapezoid-shaped device served as nonvolatile logic inputs and NDR elements were connected to output channels for large ON/OFF ratio. b. Experimentally measured output current in left (AND) and right (NAND) channel.

Table 1 Value of output current when $I_{0}$ was set to $30 \mu \mathrm{A}$

\begin{tabular}{ccc}
\hline & \multicolumn{2}{c}{ Logic Output } \\
\cline { 2 - 3 }$(\mathrm{P}, \mathrm{Q})$ & Left Channel (AND) & Right Channel (NAND) \\
\hline$(0,0)$ & $0(0.61 \mu \mathrm{A})$ & $1(29.36 \mu \mathrm{A})$ \\
\hline$(0,1)$ & $0(0.66 \mu \mathrm{A})$ & $1(29.31 \mu \mathrm{A})$ \\
\hline$(1,0)$ & $0(0.64 \mu \mathrm{A})$ & $1(29.33 \mu \mathrm{A})$ \\
\hline$(1,1)$ & $1(29.71 \mu \mathrm{A})$ & $0(0.26 \mu \mathrm{A})$ \\
\hline
\end{tabular}
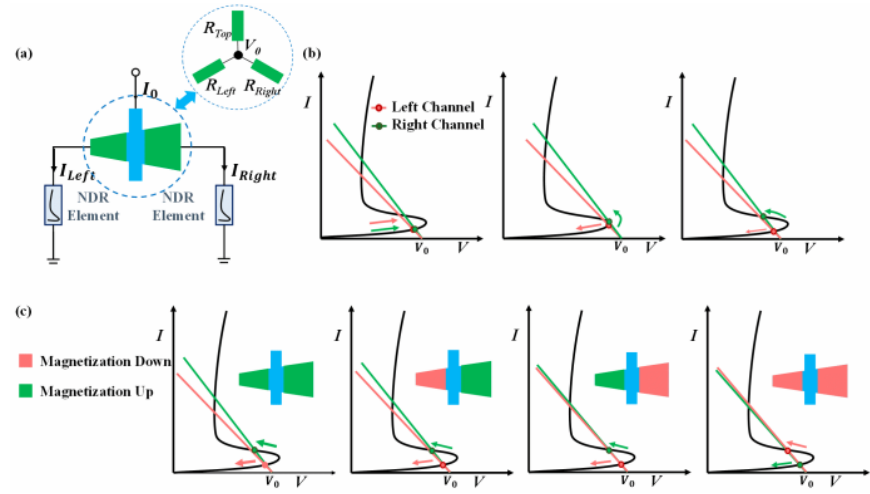

Fig. 4 Revealing the mechanism of AND/NAND logic operation with large ON/OFF ratio by load line analysis. a. Resistor network model of the three-terminal trapezoid-shape logic device b. Load line analysis when both magnetic bits were magnetized upward. As source current $\mathrm{I}_{0}$ increased, the two load lines intersected with the $\mathrm{S}$ shape $I-V$ curve of the NDR element at different region. Because of the AHE of the magnetic bits, the effective resistance of the right channel $R_{\text {Right }}$ was smaller than $R_{\text {Left. }}$ The load line of the right channel had larger slope, resulting in larger output current in the current bifurcation. c. Different magnetization states of the magnetic bits resulted in different slope of the load lines, which determined the magnitude of the output current. 
